\title{
Droplet networks with incorporated protein diodes show collective properties
}

\author{
Giovanni Maglia', Andrew J. Heron', William L. Hwang², Matthew A. Holden³, Ellina Mikhailova', \\ Qiuhong Li', Stephen Cheley ${ }^{1}$ and Hagan Bayley ${ }^{1 \star}$
}

Recently, we demonstrated that submicrolitre aqueous droplets submerged in an apolar liquid containing lipid can be tightly connected by means of lipid bilayers ${ }^{1-5}$ to form networks ${ }^{4-6}$. Droplet interface bilayers have been used for rapid screening of membrane proteins ${ }^{7,8}$ and to form asymmetric bilayers with which to examine the fundamental properties of channels and pores $^{9}$. Networks, meanwhile, have been used to form microscale batteries and to detect light ${ }^{4}$. Here, we develop an engineered protein pore with diode-like properties that can be incorporated into droplet interface bilayers in droplet networks to form devices with electrical properties including those of a current limiter, a half-wave rectifier and a full-wave rectifier. The droplet approach, which uses unsophisticated components (oil, lipid, salt water and a simple pore), can therefore be used to create multidroplet networks with collective properties that cannot be produced by droplet pairs.

To obtain directional ionic current flows in droplet networks (Fig. 1), we constructed a diode-like pore from staphylococcal $\alpha$-haemolysin ( $\alpha \mathrm{HL}$ ). $\alpha \mathrm{HL}$ forms a heptameric protein pore ${ }^{10}$ that inserts vectorially into lipid bilayers ${ }^{11}$. The crystal structure of the pore reveals a 14-stranded transmembrane $\beta$ barrel capped by an extramembraneous domain, which contains a roughly spherical cavity ${ }^{10}$ (Fig. 2a, left). The wild-type (WT) pore is a 'blank slate' for protein engineering with properties similar to those of an electrolyte-filled tube; it is weakly rectifying and weakly anion selective and gates only at extreme applied potentials of either polarity ${ }^{12}$.

$\alpha H L$ has been modified by mutagenesis or targeted chemical modification to form pores with a wide range of properties ${ }^{13-16}$, but none has exhibited sufficient rectification for our purpose. We had, however, noticed that $\alpha \mathrm{HL}$ pores with positively charged side chains projecting into the lumen of the transmembrane $\beta$ barrel tended to gate (open and close) at negative potentials. Therefore, in an attempt to obtain a fully rectifying pore, we tested an extreme version of $\alpha \mathrm{HL}$ in which seven residues were replaced with arginines $(7 \mathrm{R}-\alpha \mathrm{HL})$ to yield a heptameric pore in which 49 additional positively charged side chains were located within the barrel (Fig. 2a, right). In $1 \mathrm{M} \mathrm{KCl}, 25 \mathrm{mM}$ Tris $\mathrm{HCl}$ at $\mathrm{pH} 8.0$, $100 \mu \mathrm{M}$, in planar lipid bilayers, the $7 \mathrm{R}-\alpha \mathrm{HL}$ pore has a unitary conductance of $0.95 \pm 0.01 \mathrm{nS}(+50 \mathrm{mV}, n=8)$. The conductance of the WT pore under the same conditions is similar $(0.99 \pm 0.02 \mathrm{nS}$, $n=4)$, which suggests, surprisingly, that the drastically altered $7 \mathrm{R}-\alpha \mathrm{HL}$ pore is properly formed. The current-voltage $(I-V)$ characteristics of $7 \mathrm{R}-\alpha \mathrm{HL}$ in $1 \mathrm{M} \mathrm{KCl}$, however, showed virtually complete current rectification (Fig. 2b,c). At positive applied potentials, $7 \mathrm{R}-\alpha \mathrm{HL}$ remained in an open form with a stable steady-state current and infrequent short-lived closures of less than $10 \mu \mathrm{s}$. By contrast, at negative applied potentials, the pore was

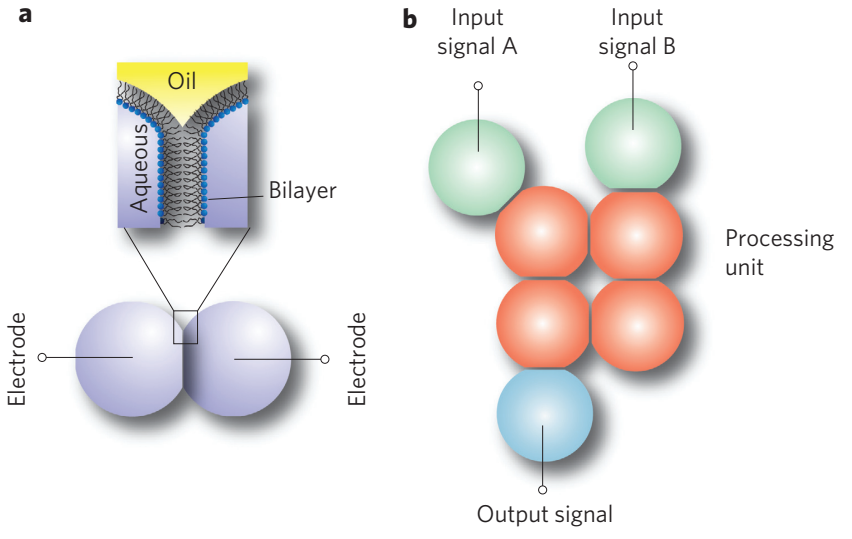

Figure 1 | Droplet interface bilayer systems. a, Two-droplet interface bilayer. Droplets ( $200 \mathrm{nl}$ ) containing $1 \mathrm{mM}$ DPhPC (as vesicles) are pipetted into hexadecane containing $5 \mathrm{mM}$ DPhPC. Over several minutes, lipid monolayers form around the droplets. Afterwards, when two droplets are brought into contact, a bilayer rapidly forms. The droplets are accessed and manipulated with $\mathrm{Ag} / \mathrm{AgCl}$ electrodes (100 $\mu \mathrm{m}$ diameter). b, Representation of a droplet network. In the hypothetical device shown, a processing unit takes two input signals and produces an output. The network exhibits a collective property; that is, the capabilities of the device are greater than those of its parts.

closed, with occasional brief current spikes ascribable to re-openings (Fig. 2b; see also Supplementary Fig. S1). By repeating a voltage-step protocol with a single $7 \mathrm{R}-\alpha \mathrm{HL}$ pore and averaging the current traces, we measured the kinetics of pore closure (Fig. 2d, bottom), which are described best by a double exponential, with a fast decay constant of $210 \pm 30 \mathrm{~ms}$, and a slower component of $1.0 \pm 0.2 \mathrm{~s}(-50 \mathrm{mV}, 1 \mathrm{M} \mathrm{KCl}, \mathrm{pH} 8.0)$. The fast decay results from the first shutting event, possibly a collapse of the $\beta$ barrel, and the long decay from occasional re-openings (see Supplementary Fig. S1).

The $7 \mathrm{R}-\alpha \mathrm{HL}$ pore exhibits almost ideal delayed (timedependent) rectification. The rectification ratio at $50 \mathrm{mV}$ (the conductance at $+50 \mathrm{mV}$ divided by the conductance at $-50 \mathrm{mV}$ ) is $g_{+50} / g_{-50}=60 \pm 10(n=6,1 \mathrm{M} \mathrm{KCl}, 25 \mathrm{mM}$ Tris HCl, pH 8.0, where the value is likely to be higher than that quoted because of the uncertainty in the leak current associated with the lipid bilayer), which is an order of magnitude higher than the rectification ratio for previously engineered biological diodes ${ }^{17,18}$. An additional advantage of the $7 \mathrm{R}-\alpha \mathrm{HL}$ pore is that it works over a range of ionic strengths (from 0.2 to $3 \mathrm{M} \mathrm{KCl}$ ), whereas all previous engineered

'Department of Chemistry, University of Oxford, Oxford OX1 3TA, UK, ${ }^{2}$ Division of Health Sciences and Technology, Harvard Medical School and Massachusetts Institute of Technology, Boston, Massachusetts 02115-5750, USA, ${ }^{3}$ Department of Chemistry, University of Massachusetts, Amherst, Massachusetts 01003, USA. *e-mail: hagan.bayley@chem.ox.ac.uk 

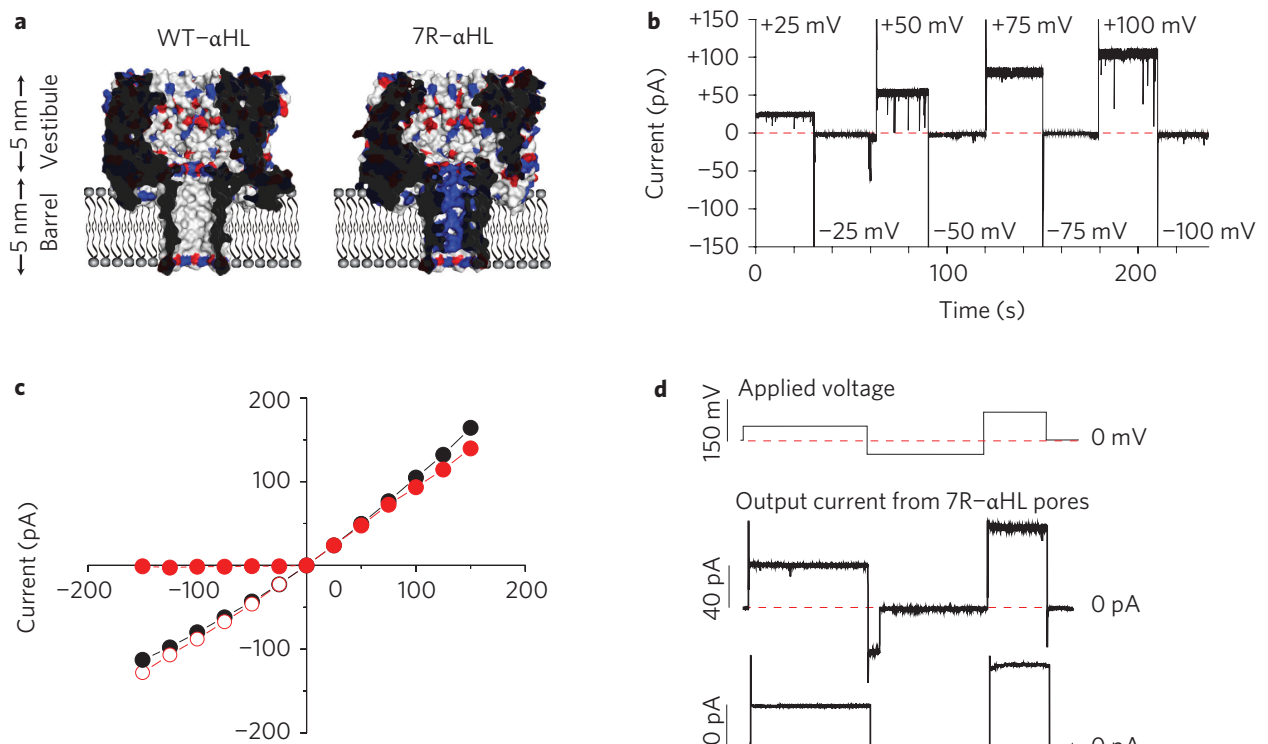

d

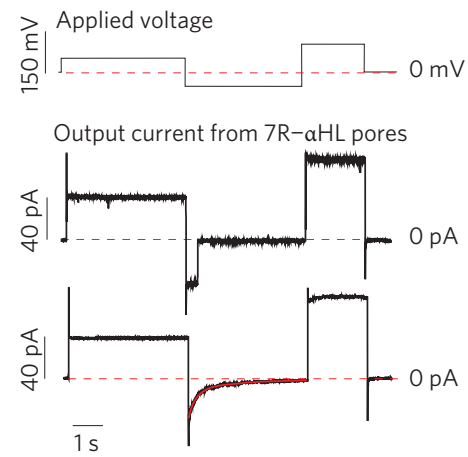

Figure 2 | Rectification of the $7 R-\alpha H L$ pore. a, Section through heptameric pores formed by WT- $\alpha H L$ (left) and $7 R-\alpha H L$ (right) (PyMOL ${ }^{T M} v 1.0$, DeLano Scientific). Negative side-chains, red; positive side-chains, blue. b, Behaviour of a single $7 R-\alpha H L$ pore at positive and negative potentials. c, $I-V$ characteristics of individual pores. WT- $\alpha \mathrm{HL}$, solid black circles; 7R- $\alpha \mathrm{HL}$ (steady-state), solid red circles; $7 \mathrm{R}-\alpha \mathrm{HL}$ (instantaneous currents, after steps in applied potential, for example, panel d), open circles. $d$, Rectification of a $7 \mathrm{R}-\alpha \mathrm{HL}$ pore under a voltage-step protocol (top): one run (middle); average of 120 consecutive runs (bottom) fit with a double exponential function (red line). Bilayer experiments were performed in $1 \mathrm{M} \mathrm{KCl}, 25 \mathrm{mM}$ Tris $\mathrm{HCl}, \mathrm{pH} 8.0,100 \mu M$ EDTA.

a
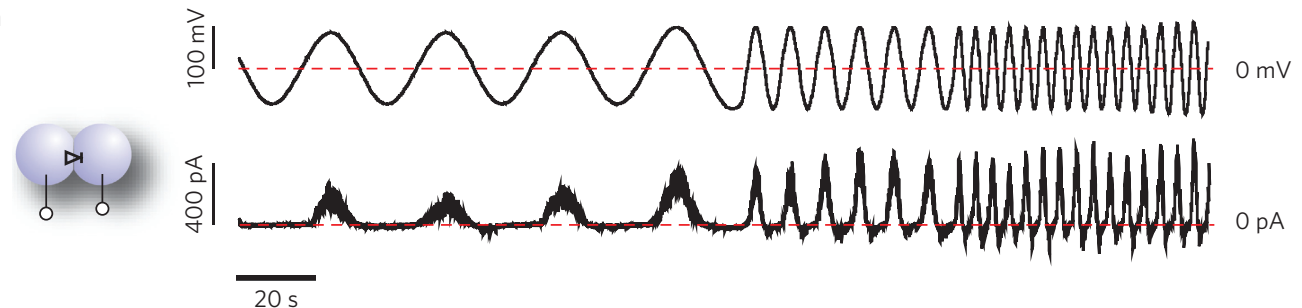

b
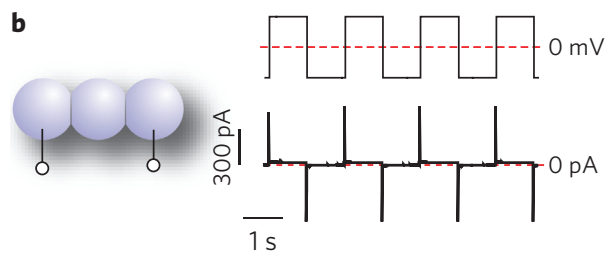

d

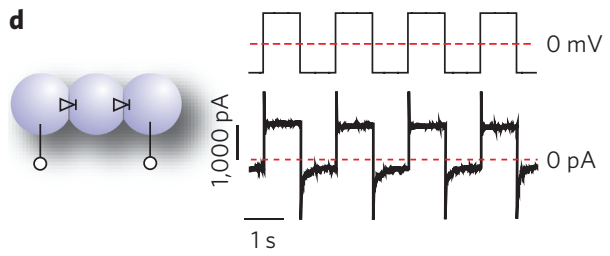

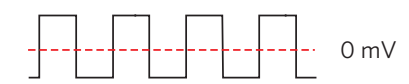
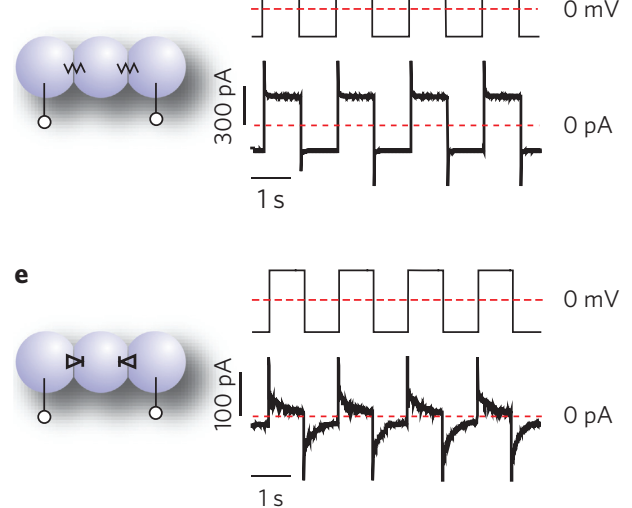

Figure 3 | Droplet networks containing WT- $\alpha H L$ and $7 R-\alpha H L$ pores. Each DIB contained up to ten pores. a, Half-wave rectification by $7 \mathrm{R}-\alpha H \mathrm{HL}$. Current (bottom) in response to a sinusoidal input $( \pm 100 \mathrm{mV})$ (top). b-e, Three-droplet networks containing WT- $\alpha \mathrm{HL}\left(\sim 50 \mathrm{pg} \mu \mathrm{l}^{-1}\right)$ or $7 \mathrm{R}-\alpha \mathrm{HL}\left(\sim 1 \mu \mathrm{g} \mu \mathrm{l}^{-1}\right)$, where output current (bottom) is in response to a square wave input of $\pm 100 \mathrm{mV}$ (top): no proteins in the network $\mathbf{b}, \mathrm{WT}-\alpha \mathrm{HL}$ in both bilayers $\mathbf{c}, 7 \mathrm{R}-\alpha \mathrm{HL}$ in both bilayers with the same orientation, creating a rectifying circuit $\mathbf{d}, 7 \mathrm{R}-\alpha \mathrm{HL}$ in both bilayers, but in opposing orientations, creating a current limiter $\mathbf{e}$, Droplet experiments were performed in $1 \mathrm{M} \mathrm{KCl}, 25 \mathrm{mM}$ Tris $\mathrm{HCl}, \mathrm{pH} 8.0,100 \mu \mathrm{M}$ EDTA.

biological and abiological diodes operate at relatively low ionic strengths (typically, less than $100 \mathrm{mM})^{17-20}$.

Recent 'nanofluidic diodes' have been prepared from protein ${ }^{17,18}$ or abiological ${ }^{19-21}$ pores by introducing static positive charges at one end of the inner surface of the pore and negative charges at the opposite end. When the pores are filled with a dilute electrolyte, they rectify ionic current by a mechanism that is thought to resemble the charge depletion that occurs at np junctions in semiconductor diodes ${ }^{22}$. The mechanism by which $7 \mathrm{R}-\alpha \mathrm{HL}$ nanopores rectify the ionic current is different. Although the nanofluidic diodes 
a

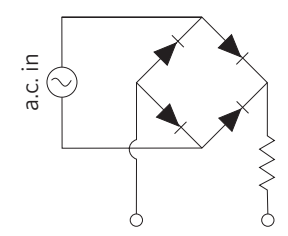

b

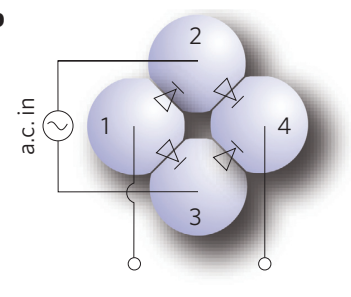

c

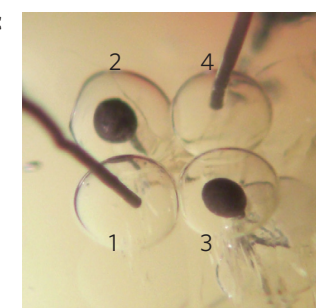

= Semiconductor diode

$\triangle 1=7 \mathrm{R}-\mathrm{\alpha HL}$ diode

d Input voltage waveform

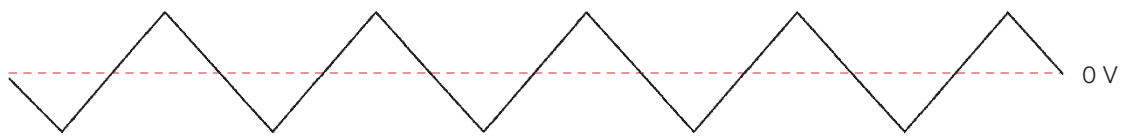

Output current from semiconductor diodes
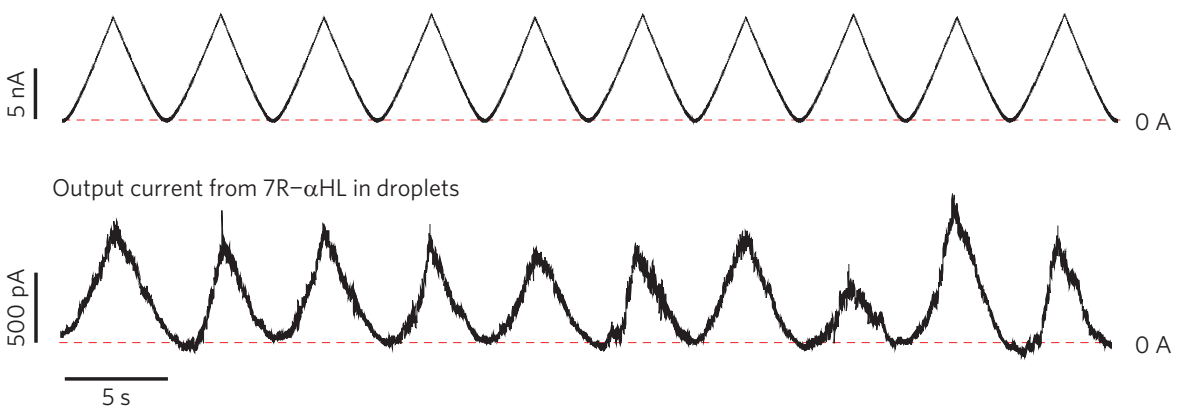

Figure 4 | Full-wave rectification in a four-droplet network. a, Bridge rectifier using four diodes to convert an alternating current into a direct current. b,c, Schematic and photograph of a four-droplet network using $7 \mathrm{R}-\alpha \mathrm{HL}$ to create a bridge rectifier. $\mathbf{d}$, The triangular $0.1 \mathrm{~Hz}$ input (top), and the current output from a semiconductor diode bridge rectifier (middle) and the 7R- $\alpha \mathrm{HL}$ network (bottom). The input waveforms were generated with a custom signal generator (see Supplementary Fig. S4). The current outputs were measured with a patch-clamp amplifier in voltage-clamp mode acting as an ammeter (no applied potential). The input waveform was $\pm 1.5 \mathrm{~V}$ for the semiconductor device, with the output measured across a $100 \mathrm{M} \Omega$ resistor. The input waveform was $\pm 100 \mathrm{mV}$ for the droplet network.

only operate efficiently when the diameter of the nanopore is comparable to the Debye length in the solution, the 7R- $\alpha \mathrm{HL}$ nanopores work efficiently at all ionic strengths tested up to $3 \mathrm{M} \mathrm{KCl}$. In addition, single-channel recording showed that at negative bias the current through the nanopore is reduced from the open value to almost zero in a single step after an exponentially distributed delay with a time constant consistent with a change in protein conformation (Fig. 2d). Further, occasional reopenings occur, which is also consistent with a conformational change such as a collapse of the transmembrane $\beta$ barrel (see Supplementary Fig. S1). If the rectification were due to the formation of a depletion zone inside the nanopore, as proposed for np junctions, the current block would be instantaneous and no re-openings would occur.

Although the response time of $7 \mathrm{R}-\alpha \mathrm{HL}$ is slow, it can be harnessed to build devices that resemble traditional electronic circuits based on semiconductors. We first used $7 \mathrm{R}-\alpha \mathrm{HL}$ to build a half-wave rectifier, which is the simplest device that converts alternating current to direct current, by permitting current flow under applied potentials of one polarity only. The device was built by reconstituting $7 \mathrm{R}-\alpha \mathrm{HL}$ into a droplet interface bilayer (DIB) and applying a sinusoidal voltage waveform with an amplitude of $\pm 100 \mathrm{mV}$ (Fig. 3a). At frequencies below $0.2 \mathrm{~Hz}$, the output current from the input sinusoidal waveform was mainly positive (Fig. 3a). The efficiency of rectification at higher frequencies is limited by the closing decay constant at negative potentials (see Supplementary Fig. S2) and a smaller contribution from the capacitance of the system (see Supplementary Fig. S5). Hence, above $0.5 \mathrm{~Hz}$, the rectification efficiency degrades substantially (see Supplementary Fig. S3).
More complex electronic functions were produced by using DIB networks. The formation of DIB networks is straightforward; droplets that have been penetrated with agarose-tipped electrodes can be moved into contact with a micromanipulator without damaging other parts of a network. The majority of insertion events arising from pores encapsulated within the droplets occur within a few minutes of bilayer formation. Therefore, the orientation of the protein diodes at each interface in a network can be controlled by selecting which droplets contain protein and the order in which the bilayers are formed. We applied a $0.5 \mathrm{~Hz}, \pm 100 \mathrm{mV}$, square voltage wave to four different three-droplet DIB networks (Fig. 3). In the protein-free network, no current was observed as expected (Fig. 3b). When WT- $\alpha \mathrm{HL}$ was incorporated into both bilayers from the middle droplet, a symmetrical output current trace that matched the input waveform was obtained (Fig. 3c). In contrast, we found that the orientation of the $7 \mathrm{R}-\alpha \mathrm{HL}$ pore in each bilayer changes the overall behaviour of the network (Fig. 3d,e). When the $7 \mathrm{R}-\alpha \mathrm{HL}$ pores in each bilayer are oriented in the same direction, current passes when the network is forward-biased, but not when it is reverse-biased, again constituting a half-wave rectifier (Fig. 3d). On the other hand, when the $7 \mathrm{R}-\alpha \mathrm{HL}$ pores in each bilayer are oriented in opposite directions, the network behaves as a current limiter, with little current passing in either direction (Fig. 3e).

The droplet technique is suitable for building networks with complex connectivity in two and potentially three dimensions to produce systems with higher-level properties. With these ideas in mind, we also built a four-droplet network, in which the compartments communicated through four DIBs. This device functioned as a bridge rectifier (Fig. 4a). Bridge rectifiers make use of four diodes 
in a configuration that provides a fixed output-voltage polarity regardless of input voltage polarity. In the four-droplet network, the correct orientation of the $7 \mathrm{R}-\alpha \mathrm{HL}$ pore in each DIB (Fig. $4 \mathrm{~b}$ ) was again achieved by controlling which droplets contained protein and in what order the droplets were connected to one another (Fig. 4c). 7R- $\alpha \mathrm{HL}$ was included in droplets 1, 2 and 3, whereas droplet 4 contained no protein. Droplets 2 and 3 were first connected to droplet 4 , and left for $\sim 10 \mathrm{~min}$, after which protein insertion ceased. Finally, droplet 1 was connected to droplets 2 and 3, and the protein in this droplet inserted into the two new interfaces (Fig. $4 \mathrm{c})$. The protein dilution $\left(\sim 1 \mu \mathrm{g} \mu \mathrm{l}^{-1}\right)$ was set so that each DIB contained 5 to 10 channels. The DIB bridge rectifier exhibited full-wave rectification of an alternating $0.1 \mathrm{~Hz}$ triangular waveform input, matching the output of its semiconductor equivalent, that is, the device permitted unidirectional current flow under applied potentials of both signs (Fig. 4d).

In the present work, we have formed small networks of droplets with the collective ability to process electrical inputs. Remarkably, these networks are formed from simple components that assemble spontaneously, namely an aqueous salt solution, a synthetic lipid and an engineered protein pore, which suggests that far more complex entities might be assembled with additional elements. In particular, the ability of the droplets to communicate throughout the networks with electrical signals suggests that the capacity to compute might be built into such systems. It follows that droplet networks might be used to build responsive drug-delivery systems that are more sophisticated than simple liposomes ${ }^{23}$ or to form interfaces between integrated circuits and living tissues ${ }^{24,25}$.

\section{Methods}

Heptameric $7 \mathrm{R}-\boldsymbol{\alpha} \mathrm{HL}$ pores. The $7 \mathrm{R}-\alpha \mathrm{HL}$ gene was constructed by cassette mutagenesis of $\alpha \mathrm{HL}-\mathrm{RL} 2$ (ref. 26) and verified by DNA sequencing. 7R- $\alpha \mathrm{HL}$ was expressed in E. coli Rosetta (DE3) pLysS cells (Merck, UK) by using a pT7 plasmid. As documented fully in the Supplementary Information, the spontaneously heptamerized form of $7 \mathrm{R}-\alpha \mathrm{HL}$ was treated with SP Sepharose resin to remove lowmolecular-weight contaminants, and further purified by gel filtration on Superdex 200 10/300GL and preparative SDS-polyacrylamide gel electrophoresis ${ }^{26}$.

Electrical recordings. Currents were measured with $\mathrm{Ag} / \mathrm{AgCl}$ electrodes by using a patch-clamp amplifier (Axopatch 200B, Axon Instruments). 1,2-diphytanoyl-snglycero-3-phosphocholine (Avanti Polar Lipids) was used to form both planar bilayers and droplet interface bilayers, and in both cases the electrolyte was $1 \mathrm{M} \mathrm{KCl}$, $25 \mathrm{mM}$ Tris $\mathrm{HCl}, \mathrm{pH} 8.0$, containing $100 \mu \mathrm{M}$ EDTA.

Received 2 March 2009; accepted 28 April 2009; published online 7 June 2009

\section{References}

1. Tsofina, L. M., Liberman, E. A. \& Babakov, A. V. Production of bimolecular protein-lipid membranes in aqueous solution. Nature 212, 681-683 (1966).

2. Funakoshi, K., Suzuki, H. \& Takeuchi, S. Lipid bilayer formation by contacting monolayers in a microfluidic device for membrane protein analysis. Anal. Chem. 78, 8169-8174 (2006)

3. Malmstadt, N., Nash, M. A., Purnell, R. F. \& Schmidt, J. J. Automated formation of lipid-bilayer membranes in a microfluidic device. Nano Lett. 6 , 1961-1965 (2006).

4. Holden, M. A., Needham, D. \& Bayley, H. Functional bionetworks from nanoliter water droplets. J. Am. Chem. Soc. 129, 8650-8655 (2007).
5. Bayley, H. et al. Droplet interface bilayers. Mol. BioSystems 4, 1191-1208 (2008)

6. Hwang, W. L., Holden, M. A., White, S. \& Bayley, H. Electrical analysis of protein pore insertion and blockade in droplet interface bilayer networks. J. Am. Chem. Soc. 129, 11854-11864 (2007).

7. Syeda, R., Holden, M. A., Hwang, W. L. \& Bayley, H. Rapid screening of blockers against a potassium channel with a droplet interface bilayer array. J. Am. Chem. Soc. 130, 15543-15548 (2008).

8. Poulos, J. L. et al. Ion channel and toxin measurement using a high throughput lipid membrane platform. Biosens. Bioelectron. 24, 1806-1810 (2009).

9. Hwang, W. L., Chen, M., Cronin, B., Holden, M. A. \& Bayley, H. Asymmetric droplet interface bilayers. J. Am. Chem. Soc. 130, 5878-5879 (2008).

10. Song, L. et al. Structure of staphylococcal $\alpha$-hemolysin, a heptameric transmembrane pore. Science 274, 1859-1865 (1996).

11. Braha, O. et al. Designed protein pores as components for biosensors. Chem. Biol. 4, 497-505 (1997).

12. Menestrina, G. Ionic channels formed by Staphylococcus aureus alpha-toxin: voltage-dependent inhibition by divalent and trivalent cations. J. Membrane Biol. 90, 177-190 (1986).

13. Bayley, H. \& Cremer, P. S. Stochastic sensors inspired by biology. Nature 413, 226-230 (2001).

14. Bayley, H. \& Jayasinghe, L. Functional engineered channels and pores. Mol. Membrane Biol. 21, 209-220 (2004).

15. Jung, Y., Cheley, S., Braha, O. \& Bayley, H. The internal cavity of the staphylococcal $\alpha$-hemolysin pore accommodates $\sim 175$ exogenous amino acid residues. Biochemistry 44, 8919-8929 (2005).

16. Wu, H.-C., Astier, Y., Maglia, G., Mikhailova, E. \& Bayley, H. Protein nanopores with covalently attached molecular adapters. J. Am. Chem. Soc. 129, 16142-16148 (2007)

17. Alcaraz, A. et al. A pH-tunable nanofluidic diode: electrochemical rectification in a reconstituted single ion channel. J. Phys. Chem. B 110, 21205-21209 (2006).

18. Miedema, H. et al. A biological porin engineered into a molecular, nanofluidic diode. Nano Lett. 7, 2886-2891 (2007).

19. Vlassiouk, I. \& Siwy, Z. S. Nanofluidic diode. Nano Lett. 7, 552-556 (2007).

20. He, Y. et al. Tuning transport properties of nanofluidic devices with local charge inversion. J. Am. Chem. Soc. 131, 5194-5202 (2009).

21. Karnik, R., Duan, C., Castelino, K., Daiguji, H. \& Majumdar, A. Rectification of ionic current in a nanofluidic diode. Nano Lett. 7, 547-551 (2007).

22. Gijs, M. A. M. Will fluidic electronics take off? Nature Nanotech. 2, 268-270 (2007).

23. Farokhzad, O. C. \& Langer, R. Impact of nanotechnology on drug delivery. ACS Nano 3, 16-20 (2009).

24. Craelius, W. The bionic man: restoring mobility. Science 295, 1018-1021 (2002).

25. Donoghue, J. P. Bridging the brain to the world: a perspective on neural interface systems. Neuron 60, 511-521 (2008)

26. Cheley, S., Braha, O., Lu, X., Conlan, S. \& Bayley, H. A functional protein pore with a 'retro' transmembrane domain. Protein Sci. 8, 1257-1267 (1999).

\section{Acknowledgements}

This work was funded by a Royal Society Wolfson Research Merit Award to H.B., the Medical Research Council and the National Institutes of Health. W.L.H. was supported by a Rhodes Scholarship.

\section{Author contributions}

G.M. designed the protein diode and E.M., Q.L. and S.C. made the protein. G.M. performed the research on planar bilayers. W.L.H., A.J.H. and M.A.H. designed the DIB networks. A.J.H. and W.L.H. created and tested the DIB networks. G.M., A.J.H., W.L.H. and H.B. wrote the paper. H.B. directed the project.

\section{Additional information}

Supplementary information accompanies this paper at www.nature.com/ naturenanotechnology. Reprints and permission information is available online at http://npg nature.com/reprintsandpermissions/. Correspondence and requests for materials should be addressed to H.B. 